\title{
Problem-Oriented Foundations of Intelligence in the Context of Superintelligence ${ }^{+}$
}

\author{
Mark Burgin \\ Department of Mathematics, University of California, Los Angeles, 520 Portola Plaza, Los Angeles, CA 90095, \\ USA; mburgin@math.ucla.edu \\ + Conference Theoretical Information Studies (TIS), Berkeley, CA, USA, 2-6 June 2019.
}

Published: 13 May 2020

\begin{abstract}
Despite the substantial interest in intelligence, both natural and artificial, and active research in this area, there is still considerable disagreement about what exactly constitutes intelligence. The problem-oriented approach to intelligence is based on evaluation of intelligence of a system by the level of problems this system is able to solve. The goal of this work is to develop a problem-oriented theory of intelligence in such a way that ecological characteristics become parameters of the problem-oriented model of intelligence. This model is constructed using mathematical theories and algorithmic reasoning. The suggested approach allows the reorganizing of the existing typologies of intelligence developed in works of different psychologists, providing better theoretical tools for intelligence measurement and evaluation.
\end{abstract}

Keywords: intelligence; superintelligence; information; problem; artificial intelligence; task; solution; measure

\section{Introduction}

Recently, the concept of superintelligence has been created, and become very popular [1,2]. However, to understand superintelligence, it is necessary at first to understand intelligence. Understanding of some systems or phenomena in science and often in mundane life comes through creation of relevant and adequate models of these systems and phenomena. For a long time, researchers ascribed intelligence only to people, and studied it in psychology, where it has become one of the most talked about subjects. In their studies of people in general, and their mental traits and behavior in particular, researchers elaborated various models of intelligence, discerning different types and kinds of intelligence of human beings and constructing measures of intelligence: analytical intelligence and analytical IQ (cf., for example, $[3,4]$ ), creative intelligence and creative IQ (cf., [5,6]), social intelligence [7], practical intelligence and practical IQ [5], and emotional intelligence and emotional IQ [8,9].

With the development of information technology, it became a focal goal to create artificial intelligence in the form of thinking machines or automata. This endeavor went into three directions: experimental studies of intelligence of people, theoretical studies of intelligence as a natural phenomenon, and the creation of more and more powerful information processing systems, such as computers, networks and other technical devices.

However, despite the substantial interest in the subject and active research, there is still considerable disagreement about what exactly constitutes intelligence. Futher, there is no standard definition of what exactly constitutes intelligence. For instance, Sternberg defines intelligence as "mental activity directed toward purposive adaptation to, selection, and shaping of real-world environments relevant to one's life." The goal of this work is to develop a problem-oriented theory of intelligence in such a way that ecological characteristics become parameters of the solvability 
model of intelligence. This model is rooted in mathematics and algorithmic reasoning. Our approach allows the reorganization of the existing typologies of intelligence developed in works of different psychologists, providing better theoretical tools for intelligence measurement and evaluation.

\section{Classification of Intelligence Models}

The general theory of information (GTI) is an innovative theoretical system with three components: the axiomatic foundation, mathematical core and functional hull [1].

The diversity of models of intelligence can be demarcated into three classes: attributive or trait-oriented, behavioral or task-oriented, and enigmatic or problem-oriented models of intelligence.

According to the attributive or trait-oriented approach, a person is intelligent if this person has definite mental or personality traits. For instance, intelligence must involve such mental abilities as logical reasoning, problem-solving and planning.

According to the problem-oriented (enigmatic) approach, a person is intelligent if this person can solve definite problems. For instance, to be intelligent, people must then be able to take what they have learned to come up with a useful solution to a problem they have noticed in the world around them.

According to the behavioral or task-oriented approach, a person is intelligent if this person can perform definite tasks.

There are natural relations between these approaches.

Proposition 1. It is possible to transform any behavioral (task-oriented) model into an enigmatic (problem-oriented) model and vice versa.

Proposition 2. It is possible to regard any behavioral (task-oriented) model as an attributive (trait-oriented) model.

Propositions 1 and 2 imply the following result:

Corollary 1. It is possible to regard any enigmatic (problem-oriented) model as an attributive (trait-oriented) model.

\section{Problem-Oriented Model of Intelligence}

According to the second ontological principle of the general theory of information, $\mathrm{O} 2$ (the General Transformation Principle), information plays the same role in the World of Structures as energy plays in the Physical (material) World [2]. Physicists studied energy much longer than researchers explored information. It makes possible learning what physicists discovered about energy and applying this knowledge to the study of information.

The history of humankind and studies of psychologists demonstrate that the concept of intelligence is a relative characteristic of different systems. How we interpret this concept depends on social and natural environment, and thus, it is ecologically reliant and contingent. This was the main obstacle in finding an encompassing, and at the same time exact, definition of intelligence.

An important property of intelligence is contextuality, which means that intelligence depends on the context in which it is considered. In particular, context includes environment, and this makes intelligence ecologically contingent. For instance, a person intelligent in jungles can be dumb in a big contemporary city and vice versa. One more important characteristic of intelligence is its graduality. It means that different people can have different levels of intelligence.

To build the solvability model of intelligence, we consider a set $\boldsymbol{P}$ of problems and two classes of systems $\mathbf{H}$ and $\mathbf{K}$.

Condition (A): For any system $T$ in $\mathbf{K}$, there is a system $A$ in $\mathbf{H}$, which can produce all results (solve all problems) that $T$ can produce (solve).

Condition (B): Any system $A$ from $\mathbf{H}$ can solve all problems from the set $\boldsymbol{P}$. 
Condition (C): For any problem $P$ from the set $\boldsymbol{P}$, there is a system $A$ in $\mathbf{H}$, which can solve it.

Lemma 1. Condition (C) follows from Condition (B).

Definition 1. (a) A class $\boldsymbol{H}$ is called complete for a class $\boldsymbol{K}$ if it satisfies Condition ( $A$ ).

(b) A system $A$ is called total for a class $K$ if the class $\{A\}$ satisfies Condition $(A)$.

(c) A class $\boldsymbol{H}$ is called complete in a class $\boldsymbol{K}$ if it satisfies Condition ( $A$ ) and $\boldsymbol{H} \subseteq \mathbf{K}$.

(d) A system A from a class $\boldsymbol{K}$ is called universal in $\boldsymbol{K}$ if the class $\{A\}$ satisfies Condition (A).

Example 1. A class $\boldsymbol{H}$ of Turing machines is complete in the class $\mathbf{T}$ of all Turing machines if it contains at least one universal Turing machine.

Example 2. A universal Turing machine is universal in the class $\mathbf{T}$ of all Turing machines.

Example 3. A class $\mathbf{H}$ of inductive Turing machines is complete in the class $\mathbf{T}$ of all inductive Turing machines if it contains at least one universal inductive Turing machine.

Lemma 2. Any class of information processing systems $\boldsymbol{K}$ is complete in itself.

Lemma 3. Any universal in $\mathbf{K}$ system is total for $\mathbf{K}$.

Proposition 3. If a class $\boldsymbol{H}$ is complete for (in) a class $\boldsymbol{K}$ and $\boldsymbol{H} \subseteq \boldsymbol{G}$, then the class $\boldsymbol{G}$ is complete for the class $\boldsymbol{K}$.

Corollary 2. If a class $\boldsymbol{H}$ contains a total for $\mathbf{K}$ system, then the class $\boldsymbol{H}$ is complete for the class $\boldsymbol{K}$.

Proposition 4. If a class $\boldsymbol{H}$ is complete for (in) a class $\boldsymbol{K}$ and $\boldsymbol{F} \subseteq \boldsymbol{K}$, then the class $\boldsymbol{H}$ is complete for the class $\boldsymbol{F}$.

Corollary 3. If a system $A$ is total for $\boldsymbol{K}$ and $\boldsymbol{F} \subseteq \boldsymbol{K}$, then the system $A$ is total for $\boldsymbol{F}$.

Corollary 4. If a class $\boldsymbol{H}$ contains a total for a class $\boldsymbol{K}$ system and $\boldsymbol{F} \subseteq \boldsymbol{K}$, then the class $\boldsymbol{H}$ is complete for the class $\boldsymbol{F}$.

Using the problem-oriented approach and taking a set of problems $\boldsymbol{P}$, which is called an intelligence parameter, we define intelligent subclasses and systems in a class $\mathbf{K}$ of systems.

Definition 2. A class $\boldsymbol{H}$ of systems is called weakly intelligent with respect to $\boldsymbol{P}$ if it satisfies Condition (B).

We denote this by $\mathbf{H}=\operatorname{Int}_{\mathrm{w}}{ }^{P}$, and take the class $\mathbf{K} \boldsymbol{P}$, that consists of all problems each of which can be solved by some system from a class $\mathbf{K}$ of systems, and systems from a class $\mathbf{H}$ can do necessary reductions of problems.

Proposition 5. A class $\boldsymbol{H}$ is complete for a class $\boldsymbol{K}$ if and only if $\boldsymbol{H}=\operatorname{Int}_{w} \mathrm{KP}$.

Corollary 5. A class $\boldsymbol{H}$ is complete in a class $\boldsymbol{K}$ if and only if $\boldsymbol{H} \subseteq \boldsymbol{K}$ and $\boldsymbol{H}=\operatorname{Int}_{w}{ }^{K \boldsymbol{P}}$.

Proposition 6. If $\boldsymbol{H}=\operatorname{Int}_{w}{ }^{P}$ and $Q \subseteq P$, then $\boldsymbol{H}=\operatorname{Int}_{w} Q$.

The mental effort of an individual who learns some portion of knowledge can be measured by time of this learning. It is also possible to define mental effort by measuring intensity of the brain activity during learning.

If the system $\boldsymbol{P}$ is determined by the class $\mathbf{K}$, we denote this by $\mathbf{H}=\operatorname{Int}_{\mathrm{w}}{ }^{P} \mathbf{K}$.

Definition 3. (a) A class $\boldsymbol{H}$ of systems is called robustly intelligent for a class $\boldsymbol{K}$ with respect to $\boldsymbol{P}$ if it satisfies Conditions $(A)$ and $(B)$. We denote this by $\boldsymbol{H}=\operatorname{Int}_{r} P \boldsymbol{K}$. 
(b) A class $\boldsymbol{H}$ of systems is called robustly intelligent in a class $\boldsymbol{K}$ with respect to $\boldsymbol{P}$ if $\boldsymbol{H} \subseteq \boldsymbol{K}$ and it satisfies Conditions $(A)$ and $(B)$. We denote this by $\mathbf{H}=\operatorname{Intrin}^{P} \boldsymbol{K}$.

In other words, a robustly intelligent with respect to $\boldsymbol{P}$ class of problems is complete and weakly intelligent.

Proposition 7. If a class $\boldsymbol{H}$ is robustly intelligent for (in) a class $\boldsymbol{K}$, i.e., $\boldsymbol{H}=\operatorname{Intr}^{P} \boldsymbol{K}\left(\boldsymbol{H}=\operatorname{Intrin}^{P} \boldsymbol{K}\right)$, and $\boldsymbol{F} \subseteq \boldsymbol{K}$, then the class $\boldsymbol{H}$ is robustly intelligent for the class $\boldsymbol{F}$., i.e., $\boldsymbol{H}=\operatorname{Int}_{r}{ }^{P} \boldsymbol{F}$.

Proposition 8. (a) If $\boldsymbol{H}=\operatorname{Int}_{r}{ }^{P} \mathbf{K}$ and $\boldsymbol{Q} \subseteq \boldsymbol{P}$, then $\boldsymbol{H}=\operatorname{In} t_{r} Q \mathbf{K}$.

(b) If $\boldsymbol{H}=$ Intrin $^{P} \boldsymbol{K}$ and $\boldsymbol{Q} \subseteq \boldsymbol{P}$, then $\boldsymbol{H}=\operatorname{Intrin} Q \mathbf{K}$.

\section{Conclusions}

The basic elements of a problem-oriented theory of intelligence are elaborated in the context of superintelligence in such a way that ecological characteristics become parameters of the problem-oriented model of intelligence. Mathematical theories and algorithmic reasoning form foundations of this theory. The suggested approach allows the reorganization of the existing typologies of intelligence developed in works of different psychologists. The next step in the development of the problem-oriented theory of intelligence presented here is to build better theoretical tools for intelligence measurement and evaluation.

Funding: This research received no external funding.

Conflicts of Interest: The author declares no conflict of interest.

\section{References}

1. Bostrom, N. Superintelligence: Paths, Dangers, Strategies; Oxford University Press: Oxford, UK, 2014.

2. Burgin, M. Swarm Superintelligence and Actor Systems. Int. J. Swarm Intell. Evol. Comput. 2017, 6, 3.

3. Spearman, C. The Abilities of Man: Their Nature and Measurement; Macmillan: New York, NY, USA, 1927.

4. Duncan, J.; Rüdiger, J.S.; Kolodny, J.; Bor, D.; Herzog, H.; Ahmed, A.; Newell, F.N.; Emslie, H. A neural basis for general intelligence. Science 2000, 289, 457-460.

5. Sternberg, R.J. Beyond IQ: A Triarchic Theory of Intelligence; Cambridge University Press: Cambridge, UK, 1985.

6. Burgin, M. Intellectual Components of Creativity; International Academy “Man in Aerospace Systems": Kiev, Ukraine, 1998. (In Ukrainian)

7. Thorndike, R.K. Intelligence and Its Uses. Harpers Mag. 1920, 140, $227-335$.

8. Mayer, J.D.; Salovey, P. The intelligence of emotional intelligence. Intelligence 1993, 17, 433-442.

9. Koonce, R. Emotional IQ, a new secret of success? Train. Dev. 1996, 50, 19-21.

(C) 2020 by the authors. Licensee MDPI, Basel, Switzerland. This article is an open access article distributed under the terms and conditions of the Creative Commons Attribution (CC BY) license (http://creativecommons.org/licenses/by/4.0/). 